EPJ manuscript No.

(will be inserted by the editor)

\title{
Theoretical Aspects of Higgs Physics at the LHC
}

\author{
Mauro Moretti ${ }^{1}$ a \\ Department of Physiscs, Ferrara University and INFN Ferrara \\ Received: date / Revised version: date
}

\begin{abstract}
The strategies recently developed to study Higgs boson properties at the LHC are reviewed. It is shown how to obtain model-independent determinations of couplings to fermions and gauge bosons by exploiting different production and decay channels. We consider the case of Weak Boson Fusion Higgs production with $H \rightarrow b \bar{b}$
\end{abstract}

PACS. 14.80.Bn Standard-Model Higgs boson - 13.85.Hd Inelastic Scattering: many particle final state

\section{Introduction}

The LHC will allow the discovery of the Higgs boson and the study of its mass, width and couplings to fermions and gauge bosons. While the decay channels $H \rightarrow \gamma \gamma$ and $H \rightarrow Z Z^{(*)} \rightarrow 4 l$ will allow a direct mass measurement at the $0.1 \%$ level over a wide range of masses 1 , the total width can only be determined with about $10 \%$ accuracy by direct measurement of the decay $H \rightarrow Z Z^{(*)} \rightarrow 4 l$ for $m_{H}>200 \mathrm{GeV}$, (the Higgs width for lower Higgs masses being too small with respect to the detector resolution). An indirect measurement of the total width can be performed also in the low mass region by exploiting the available production and decay mechanisms at the LHC. Several studies have been performed to improve on the strategy originally proposed in ref. 2 for the determination of the Higgs boson properties. We will briefly review the progress recently made in this field. The main focus will be on the mass window $115-200 \mathrm{GeV}$, the one preferred by electroweak precision data and by supersymmetry.

\section{Theoretical calculations}

In order to disentangle a signal from backgrounds, a good understanding of uncertainties in theoretical predictions is necessary. The QCD corrections, at least at next-toleading order (NLO), are known for all production channels, the most recent calculations being the NNLO calculation $^{1}$ for the gluon fusion process in the limit of heavy top-quark mass [3 4] and the NLO corrections for the process $p p / p \bar{p} \rightarrow t \bar{t} H+X$ [5]. At present, the uncertainties arising from QCD uncertainties (combining the residual

\footnotetext{
a I acknowledge the financial support of the European Union under the contract HPRN-CT-2000-00149

1 The $\mathrm{K}$ factor for the NLO contribution is 2 making desirable the knowledge of NNLO contribution
}

scale dependence with the error from parton distribution functions) can be estimated to be $\pm 20 \%$ for gluon fusion, $\pm 5 \%$ for $\mathrm{WBF}, \pm 10 \%$ for associated production.

Concerning the backgrounds, several processes with low final state parton multiplicity (corresponding to important irreducible backgrounds) are available at NLO, namely $q \bar{q} \rightarrow \gamma \gamma$ [6], gg $\rightarrow \gamma \gamma$ [7], $p p(\bar{p}) \rightarrow W b \bar{b}, p p(\bar{p}) \rightarrow$ $Z b \bar{b}[$ ] $, p p(\bar{p}) \rightarrow W j j, p p(\bar{p}) \rightarrow Z j j[9], p p(\bar{p}) \rightarrow V V$ [10] and QCD $H+j j$ production via gluon fusion 11. Some of these calculations are already implemented in NLO Monte Carlo programs. In the case of multiparton final states, the methods developed for NLO calculations cannot be applied, because of the complexity of the calculations for processes with many external legs. Recently some effort has been devoted to the realization of LO Monte Carlo event generators based on exact matrix element calculations, interfaced to the shower evolution Monte Carlo programs producing the real final state hadrons [12,13, 14, 15. 16.

\section{Higgs couplings to fermions and gauge bosons}

In principle, the Higgs coupling to a given fermion family $f$, could be obtained from the following relation:

$$
R(H \rightarrow f \bar{f})=\int L d t \cdot \sigma(p p \rightarrow H) \cdot \frac{\Gamma_{f}}{\Gamma},
$$

where $R(H \rightarrow f \bar{f})$ is the Higgs production rate in a given final state, $\int L d t$ is the integrated luminosity, $\sigma(p p \rightarrow H)$ is the Higgs production cross section, while $\Gamma$ and $\Gamma_{f}$ are the total and partial Higgs widths respectively. Aiming at model-independent coupling determinations, one needs to consider ratios of couplings, which are experimentally accessible through the measurements of ratios of rates for different final states, because the total Higgs cross-section 
and width cancel in the ratios (as well as the luminosity and all the QCD uncertainties related to the initial state).

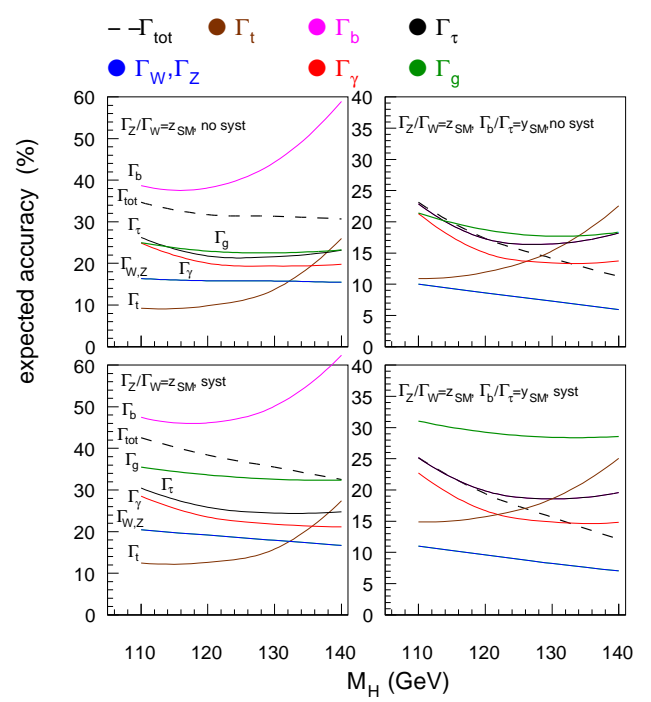

Fig. 1. Relative accuracy (\%) on the individual rates $\Gamma_{i}$ expected at the LHC (from ref. [28]). See the text for a detailed description of the panels.

Up to now detailed studies on signal and backgrounds for several channels have been performed, namely $g g \rightarrow$ $H,(H \rightarrow \gamma \gamma, Z Z, W W)$ 1] 17] 19, $q q \rightarrow q q H,(H \rightarrow$ $\gamma \gamma$,

$\tau \tau, W W)$ 20 21 22 24, $p p \rightarrow t \bar{t} H,(H \rightarrow b \bar{b}, W W, \tau \tau)[2$ 26 27,28] and $p p \rightarrow W H, H \rightarrow b \bar{b}$ 29. Each process depends on two ${ }^{2}$ Higgs couplings, one from the Higgs boson production and one from the Higgs boson decay.

Therefore, every production and decay channel provides a measurement of the ratio $Z_{j}^{(i)}=\Gamma_{i} \Gamma_{j} / \Gamma$, where $i=g, W, t$ indicates the particles involved in the production process while the index $j=b, \tau, W, Z, g, \gamma$ refers to the decay process. In case of $m_{H}<140 \mathrm{GeV}$, the above mentioned channels allow to express the individual rates $\Gamma_{t}, \Gamma_{b}, \Gamma_{\tau}, \Gamma_{W}, \Gamma_{g}$ and $\Gamma_{\gamma}$ as functions of the observables $Z_{j}^{(i)}$ and of the total Higgs width $\Gamma$ [28. With the assumption that the total width is saturated by the known channels $\Gamma=\Gamma_{b}+\Gamma_{\tau}+\Gamma_{W}+\Gamma_{Z}+\Gamma_{g}+\Gamma_{\gamma}$ (otherwise new processes would be observed independently of any precision study), an expression for $\Gamma$ can be obtained in terms of the measured quantities $Z_{j}^{(i)}$ [28]. Figure 128 summarizes the relative accuracy on the individual rates $\Gamma_{i}$ expected in the model-independent scenario as well as in a scenario with $\Gamma_{b} / \Gamma_{\tau}$ fixed to its $\mathrm{SM}$ value, assuming a total integrated luminosity of $200 \mathrm{fb}^{-1}$. The upper plots show the accuracies obtained without including any theoretical error, while the lower plots show the same accuracies including a systematic theoretical error of $20 \%$ for the $g g \rightarrow H$ channel, of $5 \%$ for the $q q \rightarrow q q H$, and

\footnotetext{
2 This is not true in the case of the weak boson fusion channel where one needs to assume that the ratio of $H W W$ and $H Z Z$ is the same as in the SM
}

of $10 \%$ for the $p p \rightarrow t \bar{t} H$. As can be seen, the total Higgs width can be indirectly determined in the low mass region with a precision of the order of $30 \%$ in a modelindependent way while the Higgs couplings can be determined with accuracies between $7 \%$ and $25 \%$. In the case of $140<m_{H}<200 \mathrm{GeV}$, the gluon fusion, weak boson fusion and $t \bar{t} H$ associated production processes, with the Higgs boson decaying only to gauge bosons, allow an indirect determination of $\Gamma_{W}$ and $\Gamma$ with a precision of the order of $10 \%$ [2,30]. In this Higgs mass range, however, there is no handle to study the Higgs Yukawa coulings to $b$ quarks and $\tau$ leptons. The assumption $\Gamma_{Z} / \Gamma_{W}=z_{S M}$ can be tested at the $20-30 \%$ level, for $m_{H}>130 \mathrm{GeV}$, by measuring the ratio $Z_{Z}^{(g)} / Z_{W}^{(g)}$ [30], and it can even be tested with the same level of accuracy for lower Higgs boson masses by comparing the two ratios $Z_{b}^{(W H)} / Z_{b}^{(t)}$ and $Z_{\tau}^{(W)} / Z_{\tau}^{(t)}$ [28]. For $m_{H}>140 \mathrm{GeV}$, with luminosities of the order of $300 \mathrm{fb}^{-1}$, the ratio $\Gamma_{t} / \Gamma_{g}$ can be tested in a model-independent measuring $Z_{W}^{(t)} / Z_{W}^{(g)}$ [27].

\section{$4 \mathrm{H} \rightarrow \mathrm{b} \overline{\mathrm{b}}$ via Weak Boson Fusion}

To improve the analysis of the $H b \bar{b}$ Yukawa coupling, one can consider the decay of an Higgs, produced via Weak Boson Fusion, into $b \bar{b}$ pairs 31 .

Signal and background event estimates are based on a leading order partonic calculation of the matrix elements (ME) obtained with the event generator ALPGEN [12]. 25|The background sources considered include:

1. QCD production of $b \bar{b} j j$ final states, where $j$ indicates a jet originating from a light quark $(u, d, s, c)$ or a gluon;

2. QCD production of $j j j j$ final states;

3. associated production of $Z^{*} / \gamma^{*} \rightarrow b \bar{b}$ and light jets

along with multiple interaction events $(p p \oplus p p, p p \oplus p p \oplus$ $p p \ldots$..) giving rise to final states of the kind $b \bar{b} j j$ and $j j j j$. In order to satisfy the requirements of optimization of the signal significance $(S / \sqrt{B})$ and compatibility with trigger and data acquisition constraints, different selection criteria have been considered. The sensitivity can be as large as 5 for Higgs masses close to the exclusion limit given by LEP searches but the ratio $S / B$ is only a fraction of a percent. This implies that the background will have to be known with accuracies at the permille level. The background should therefore be determined entirely from data. The large rate of $b \bar{b} j j$ from single and multiple interactions and the smoothness of their mass distribution will allow to estimate their size with enough statistical accuracy, without significant systematic uncertainties.

The situation is different in the case of the backgrounds from the tails of the $Z$ decays. The $Z$ mass peak is sufficiently close to $m_{H}$ to possibly distort the $m_{b b}$ spectrum and spoil the ability to accurately reconstruct the noise level from data. These backgrounds rates are at most comparable to the signal at low $m_{H}$. A $10 \%$ determination of these final states, which should be easily achievable using 
the $\left(Z \rightarrow \ell^{+} \ell^{-}\right) j j$ control sample and folding in the detector energy resolution for jets, should therefore be sufficient to fix these background with the required accuracy.

Concerning the multiple interactions, in the simplest case of two overlapping events $(p p \oplus p p)$, there are four possible (including mistagging effects) combinations of events leading to a $b \bar{b} j j$ background. The largest contribution arises from $\left(j j_{b}\right) \oplus\left(j j_{b}\right)$ events, where the $b \bar{b}$ mass spectrum has a broad peak in the middle of the signal region. The absolute rate of these events can be determined if the distribution of the beam-line $z$ vertex separation between the two overlapping events can be determined with a resolution of of $5-10 \mathrm{~mm}$. The nunber of these events is significantly lowered using the higher threshold of $80 \mathrm{GeV}$ for the forward jets.

Table 1 summarizes the accuracy reachable in the $\mathcal{B}(H \rightarrow$ $b \bar{b})$ and in the $H b \bar{b}$ Yukawa coupling for the case of two different event selections (described in detail in ref. 31]), assuming that the coupling $H W W$ is the one predicted by the Standard Model or determined in other reactions studied in the literature. An integrated luminosity of $600 \mathrm{fb}^{-1}$ is considered. The $H \rightarrow b \bar{b}$ decay in the WBF channel

\begin{tabular}{lllll}
\hline & $m_{H}(\mathrm{GeV})$ & 115 & 120 & 140 \\
\hline (a) & $\delta \Gamma_{b} / \Gamma$ & 0.33 & 0.35 & 0.71 \\
& $\delta y_{H b b} / y_{H b b}$ & 0.58 & 0.51 & 0.56 \\
\hline \multirow{2}{*}{$(b)$} & $\delta \Gamma_{b} / \Gamma$ & 0.20 & 0.19 & 0.37 \\
& $\delta y_{H b b} / y_{H b b}$ & 0.36 & 0.30 & 0.29 \\
\hline
\end{tabular}

Table 1. The statistical significance of the determination of the branching ratio $\Gamma_{b} / \Gamma$ and of the $b$-quark Yukawa coupling in the configurations (a) and (b) (see ref. 31 for a description of the event selections), for an integrated luminosity of $600 \mathrm{fb}^{-1}$. The $p_{\mathrm{T}}^{j}$ cut on jets is $p_{\mathrm{T}}^{j}>60 \mathrm{GeV}$. The case of $p_{\mathrm{T}}^{j}>80 \mathrm{GeV}$ [31] doesn't affect sizeably the results. The probability to mistag a light-jet as b-jet is assumed to be 0.01 .

could be used together with other processes already examined in the literature for a model independent determination of the ratio of Yukawa couplings $y_{H b b} / y_{H \tau \tau}$ 32.

As a conclusion of the analysis presented in ref. 31, the $H \rightarrow b \bar{b}$ channel produced in association with two jets is suggested as an additional channel to be exploited for the measurements of the Higgs couplings to fermions.

\section{Summary}

During the last few years there has been a dramatic improvement in both theoretical and experimental studies of several Higgs boson production and decay channels at the LHC. A strategy has been designed to study, in a model-independent way, the Higgs couplings to fermions and bosons, which allows also, with little theoretical assumption, an indirect determination of the total Higgs width. The main results of a very recent analysis of the $H \rightarrow b \bar{b}$ channel in Weak Boson Fusion production have been reviewed, pointing out its importance for the determination of the $H b \bar{b}$ Yukawa coupling.

\section{Acknowledgements}

I wish to acknowledge M.L. Mangano, F. Piccinini, R. Pittau and A. Polosa for fruitful collaboration. I wish to thank the organizers for the kind invitation and for the pleasant atmosphere during the Workshop.

\section{References}

1. ATLAS Collaboration, Tech. Rep. CERN/LHCC/99-15, CERN, 1999.

2. R. Kinnunen et al., Phys. Rev. D62 (2000) 013009.

3. M. Grazzini, hep-ph/0209302 S. Catani, D. de Florian and M. Grazzini, JHEP 0105 (2001) 025; and JHEP 0201 (2002) 015; G. Bozzi, S. Catani, D. de Florain, M. Grazzini, hep-ph/0302104 S. Catani, D. de Florain, M. Grazzini, P.Nason, hep-ph/0306211

4. W.B. Kilgore, these proceedings; R.V. Harlander and W.B. Kilgore, Phys. Rev. D64 (2001) 025; Phys. Rev. Lett. 88 (2002) 201801; C. Anastasiou and K. Melnikov, Nucl. Phys. B 646 (2002) 220; V. Ravindran, J. Smith, W.L. Van Neerven, hep-ph/0302135 .

5. W. Beenakker et al., Phys. Rev. Lett. 87 (2001) 201805; S. Dawson and L. Reina, Phys. Rev. Lett. 87 (2001) 201804; S. Dawson, L. Reina and D. Wackeroth, Phys. Rev. D65 (2002) 053017; S. Dawson, C. Jackson, L.H. Orr, L. Reina and D. Wackeroth, hep-ph/0305087

6. T. Binoth et al., Eur. Phys. J. C16 (2000) 311; hep-ph/0203064

7. Z. Bern, L. Dixon and C. Schmidt, hep-ph/0206194

8. R.K. Ellis and S. Veseli, Phys. Rev. D60 (1999) 011501; J. Campbell, hep-ph/0105226

9. J. Campbell and R.K. Ellis, hep-ph/0202176

10. S. Frixione and B.R. Webber, hep-ph/0204244

11. V. Del Duca et al., Nucl. Phys. B616 (2001) 367.

12. M.L. Mangano et al., hep-ph/0206293

13. A. Pukhov et al., hep-ph/9908288

14. T. Ishikawa et al., MINAMI-TATEYA Group Coll., KEK92-19;

15. T. Stelzer and W.F. Long, Comput. Phys. Commun. 81 (1994) 357; F. Maltoni and T. Stelzer, hep-ph/0208156

16. B.P. Kersevan and E. Richter-Was, hep-ph/0201302

17. CMS Collaboration, Tech. Rep. CERN/LHCC/94-38, CERN, 1994.

18. M. Dittmar, and H.K. Dreiner, Phys. Rev. D55 (1997) 167; hep-ph/9703401

19. D. Denegri et al., hep-ph/0112045

20. D. Rainwater and D. Zeppenfeld, JHEP 12 (1997) 005.

21. D. Rainwater, D. Zeppenfeld and K. Hagiwara, Phys. Rev. D59 (1999) 014037.

22. T. Plehn, D. Rainwater and D. Zeppenfeld, Phys. Rev. D61 (2000)

23. D. Rainwater and D. Zeppenfeld, Phys. Rev. D60 (1999) 113004.

24. N. Kauer et al., Phys. Lett. B503 (2001) 113.

25. E. Richter-Was and M. Sapinski, Acta Phys. Polon. B30 (1999) 1001.

26. V. Drollinger, T. Müller and D. Denegri, hep-ph/0111312

27. F. Maltoni, D. Rainwater and S. Willenbrock, hep-ph/0202205

28. A. Belyaev and L. Reina, hep-ph/0205270

29. V. Drollinger, T. Müller and D. Denegri, hep-ph/0201249 
30. D. Zeppenfeld, hep-ph/0203123

31. M.L. Mangano et al., hep-ph/0210261

32. D. Rainwater, D. Zeppenfeld and K. Hagiwara, Phys. Rev. D59, 014037 (1999). 